\title{
Editorial
}

Journal of Innate

Immunity

\section{Natural Killer Cells: Biology, Physiology and Medicine - Part 2}

\author{
Yenan T. Bryceson Hans-Gustaf Ljunggren \\ Center for Infectious Medicine, Department of Medicine, Karolinska Institutet, Karolinska University Hospital, \\ Stockholm, Sweden
}

As we described in an editorial in the last issue of the Journal of Innate Immunity (Vol. 3, No. 3, 2011), a number of groups in the beginning of the 1970s observed unexpected spontaneous cytotoxic activities among lymphocytes. This 'annoying' background phenomenon led to the identification of a new subpopulation of lymphocytes $[1,2]$ named 'natural killer' or briefly ' $\mathrm{NK}$ ' cells by Kiessling et al. [2]. While initially engaging only a small community of scientist, the first insights into the molecular specificity of NK cells [3-5], insights into their role in controlling virus and intracellular bacteria infections as well as certain tumors and insights into their ability to regulate other immune cells [6] have put NK cells in the front seat of modern immunology. New functions of the cells are now rapidly being described (e.g. their possible role in controlling T-cell and macrophage homeostasis [7] and the possibilities of using them in therapeutic settings of human cancer [8]). As stated, the recent identification of adaptive immune cell features of NK cells is a hot topic' at the moment in the NK cell community [9]. Clearly, the study of NK cells now engages a large community of scientists working in diverse areas of NK cell research in experimental model systems as well as in humans [10].

In this issue of the Journal of Innate Immunity, as well as in the last issue of the journal, a series of updated review articles on NK cells are published. They summarize some of the most recent activities within this growing field of research. Several leading scientists have made seminal contributions to these two issues. In Part 1 (Vol. 3, No. 3, 2011), we published a series of reviews related to the molecular specificity of NK cells, including studies of their receptors and receptor signaling. Other reviews discussed NK cell interactions with other immune cells and their responses to infections. In Part 2 (this issue), we publish reviews related to the life span of NK cells, as well as their development, differentiation and senescence. Furthermore, a series of reviews on NK cell interactions with tumor cells, as well as reviews describing new insights into hematopoietic stem cell transplantation (HSCT) where NK cells may contribute to human tumor eradication, are published. Finally, articles that cover the role of NK cells in organ transplant rejection and inflammatory disease settings are included.

In the first review, Sitnicka [11] nicely presents the progress made thus far in the dissection of the developmental stages of mouse NK cells, from HSC in bone marrow to lineage-committed NK cells. Linked to this topic, Gayoso et al. [12] discuss how aging in humans affects NK cells. They interestingly describe altered proportions of NK cell subsets in elderly people and discuss how these alterations may explain the functional changes of NK cells associated with aging [12]. As outlined above, one of the best-described functions of NK cells is the ability to kill virus-infected and transformed cells. Hence, evolutionary pressures on viruses over million of years as well as on tumor cells in the local host-tumor 'microenvironment' have led to mechanisms promoting escape from NK cells. In this context, Groth et al. [13] nicely review such immune escape mechanisms serving to overcome NK cell tumor and viral immunosurveillance. In the next

\section{KARGER}

Fax +4161306 1234

E-Mail karger@karger.ch

www.karger.com
(C) 2011 S. Karger AG, Basel

$1662-811 X / 11 / 0034-0327 \$ 38.00 / 0$

Accessible online at:

www.karger.com/jin
Dr. Yenan T. Bryceson

Center for Infectious Medicine, Department of Medicine

Karolinska Institutet, Karolinska University Hospital Huddinge

SE-14186 Stockholm (Sweden)

Tel. +46858581158, E-Mail yenan.bryceson@ki.se 
review, the molecular details underlying NK cell activation during tumor progression, the influence of the tumor microenvironment on NK cells and the mechanisms that interfere with their effector function in cancer patients are reviewed by Stojanovic and Cerwenka [14]. They summarize our current knowledge of NK cells in solid tumors and discuss the design of NK cell-based therapies. One type of tumor that over the years has attracted significant interest with respect to NK cells is melanoma. Morgado et al. [15] provide a review on the recent advances in the study of melanoma cell susceptibility to NK cell-mediated lysis and describe how multiple receptor-ligand interactions participate in melanoma cell recognition and elimination. Still on the subject of NK cells and tumors, Placke et al. [16] discuss how platelets affect NK-tumor cell interactions. As stated by the authors, while NK cell interactions with other cells such as dendritic cells or monocytes/macrophages are well characterized, the knowledge regarding their crosstalk with platelets is much more limited. Placke et al. [16] provide a rationale for such studies and review the available data regarding tumor-platelet-NK cell interactions, with a particular emphasis on metastatic tumor spread. One of the therapeutic areas where NK cells have been implicated is HSCT. In this context, Nguyen et al. [17] provide an excellent review on the role of NK cells in HSCT. As Nguyen et al. argue, NK cells may play a crucial role in achieving successful transplantation in settings of HSCT. In contrast to T cells, NK cells recover quickly after HSCT and may mediate strong graft-versus-leukemia effects without inducing graft-versus-host disease. This is a key issue discussed is settings of HSCT and includes issues related to the identification of optimal HSC donors [17]. Finally, two reviews are included on the topics of NK cells in solid organ transplantation and NK cells in human psoriasis. Villard [18] reviews the role of NK cells in the context of rejection of solid organ and tissue transplants. Clearly, this is an area of research where more knowledge is needed. Tobin et al. [19] set out to discuss psoriasis, one of the most common immune-mediated disorders. In this disease, NK cells as well as other cells, such as NKT cells, have been said to have unique roles; current knowledge on this topic is discussed.

Clearly, as depicted in the represented series of reviews, research on NK cells is rapidly expanding. As we mentioned in the last issue of the Journal of Innate Immunity, it is our hope that the interested reader will become updated with regard to some of the very many interesting topics of research that the NK cell field has to offer. Once more, enjoy!

\section{References}

1 Herberman RB, Nunn ME, Lavrin DH: Natural cytotoxic reactivity of mouse lymphoid cells against syngeneic acid allogeneic tumors. 1. Distribution of reactivity and specificity. Int J Cancer 1975;16:216-229.

-2 Kiessling R, Klein E, Wigzell H: 'Natural' killer cells in the mouse. 1. Cytotoxic cells with specificity for mouse Moloney leukemia cells: specificity and distribution according to genotype. Eur J Immunol 1975;5: 112-117.

>3 Kärre K, Ljunggren HG, Piontek G, Kiessling R: Selective rejection of H-2-deficient lymphoma variants suggests alternative immune defense strategy. Nature 1986;319:675-678.

4 Ljunggren HG, Kärre K: In search of the 'missing self': MHC molecules and NK cell recognition. Immunol Today 1990;11:237244.

5 Bryceson YT, Ljunggren HG, Long EO: Minimal requirement for induction of natural cytotoxicity and intersection of activation signals by inhibitory receptors. Blood 2009; 114:2657-2666.

6 Vivier E, Tomasello E, Baratin M, Walzer T, Ugolini S: Functions of natural killer cells. Nat Immunol 2008;9:503-510.
7 Bryceson YT, Rudd E, Zheng C, Edner J, Ma D, Wood SM, Bechensteen AG, Boelens JJ, Celkan T, Farah RA, Hultenby K, Winiarski J, Roche PA, Nordenskjöld M, Henter JI, Long EO, Ljunggren HG: Defective cytotoxic lymphocyte degranulation in syntaxin-11 deficient familial hemophagocytic lymphohistiocytosis 4 (FHL4) patients. Blood 2007; 110:1906-1915.

8 Ljunggren HG, Malmberg KJ: Prospects for the use of NK cells in immunotherapy of human cancer. Nat Rev Immunol 2007;7:329339.

-9 Vivier E, Raulet DH, Moretta A, Caligiuri MA, Zitvogel L, Lanier LL, Yokoyama WM, Ugolini S: Innate or adaptive immunity? The example of natural killer cells. Science 2011; 331:44-49.

10 Colonna M, Jonjic S, Watzl C: Natural killer cells: fighting viruses and much more. Nat Immunol 2011;12:107-110.

11 Sitnicka E: Early cellular pathways of murine NK cell development. J Innate Immun 2011; 3:329-336

12 Gayoso I, Sánchez-Correa B, Campos C, Alonso C, Pera A, Casado JG, Morgado S, Tarazona R, Solana R: Immunosenescence of human NK cells. J Innate Immun 2011; $3: 337-343$.
13 Groth A, Klöss S, Pogge von Strandmann E, Köhl U, Koch J: Tumor and viral immune escape mechanisms which overcome NK cell dependent immunosurveillance. J Innate Immun 2011;3:344-354.

-14 Stojanovic A, Cerwenka A: NK cells and solid tumors. J Innate Immun 2011;3:355364.

- 15 Morgado S, Sanchez-Correa B, Casado JG, Duran E, Gayoso I, Solana R, Tarazona R: NK cell recognition and killing of melanoma cells is controlled by multiple activating receptor-ligand interactions. J Innate Immun 2011;3:365-373.

16 Placke T, Kopp HG, Rainer-Salih H: Modulation of NK cell anti-tumor reactivity by platelets. J Innate Immun 2011;3:374-382.

17 Nguyen S, Béziat V, Roos-Weil D, Vieillard V: Role of NK cells in hematopoietic stem cell transplantation: myth or reality. J Innate Immun 2011;3:383-394.

18 Villard J: The role of natural killer cells in solid organ and tissue transplantation. J Innate Immun 2011:3:395-402.

19 Tobin AM, Lunch L, Kirby B, O’Farrelly C: Natural killer cells and natural killer T cells in psoriasis. J Innate Immun 2011;3:403-410. 